\title{
ARCHIVES OF DISEASE IN CHILDHOOD
}

The Fournal of the Royal College of Paediatrics and Child Health

\section{Annotations}

\section{Attention deficit hyperactivity disorder}

An enduring pattern of restlessness, poorly focused attention, and impulsive excitability that is more or less constant across different situations and impairs a child's functioning has been recognised by paediatricians since Still described it nearly a century ago. ${ }^{1}$ Treatment of such a constellation with stimulant medication has been documented for over half a century. ${ }^{2}$ So why is there currently such a fuss? One answer might be that new labels have given the impression that there is a new understanding of the fundamental deficit.

Historically, professionals have focused on different components of the pattern. Hyperactivity (hyperkinesis) was considered the dominant feature until the 1970s when a shift in research interest to inattention led to the adoption in 1980 of the term attention deficit disorder. This could exist with or without hyperactivity (ADD $\pm \mathrm{H}$ ) in the American Psychiatric Association's Diagnostic and statistical manual (DSM-III). When revised in DSM-III-R, this became attention deficit hyperactivity disorder (ADHD), a term which persists in the current DSM-IV. The WHO International classification of diseases (ICD) retained the emphasis on hyperactivity in both its 9 th and 10 th revision. The term hyperkinetic disorder (HD) is current in ICD-10. For both ADHD and HD the clinical elements required for the definition are essentially behavioural, in spite of the attention deficit concept.

Research on inattentiveness has been of good quality but eventually unsatisfactory as different measures of attention have yielded inconsistent results, and no diagnostic test of inattention has emerged as clinically valid. Conceptually the field has moved on. The work of Sergeant's group in emphasising abnormalities in state regulation and allocation of cognitive effort, ${ }^{3}$ ideas arising from the concept of cognitive executive function (planning, organising, self monitoring ${ }^{4}$ ), and interest in how children stop themselves responding or learn to be patient has generally led to a recent burst of research on dysfunctions of response inhibition. In particular the concept of aversion to delay has been explored by Sonuga-Barke et $a l^{5}$ and the field of academic inquiry has moved away from attention itself even though it seems unlikely that delay aversion can explain everything. ${ }^{6}$ Attention remains a crucial issue in the concept of a developmental attention disorder without hyperactivity (ADD without $\mathrm{H}$ in DSM-III terms or ADHD predominantly inattentive type in DSM-IV) and this just might be a different condition altogether in spite of current classification. Hard scientific evidence for it as a valid clinical entity is sparse in spite of enthusiastic advocates.

The concept of a straightforward deficit in sustained attention associated with hyperactivity has run its course as far as ADHD is concerned and the term is therefore somewhat misleading. In view of the fact that hyperactivity itself is a powerful predictor of future social dysfunction, a return to the terminology of primary disorders of hyperactivity might be welcome. However, there is no objective diagnostic test for hyperactivity either, and attempts to measure overall rates of activity or rapidity of individual movements have been unsuccessful in discriminating a clinical population. Current tests of response inhibition have either suffered a similar fate or are in their infancy. ${ }^{6}$

Furthermore, neuroimaging, biochemistry, and psychometry have all yielded information which, although of great interest, is not yet sufficiently sensitive or specific to be of routine use. The diagnosis remains a clinical one and based on behavioural criteria. Not much has changed in clinical practice in spite of different nomenclature and intense research activity.

The approach taken by DSM-IV and by the research edition of ICD-10 is to require a certain number of behavioural elements to be present. They must be present in more than one situation, have been manifest since an early age, and impair the child's development or social functioning. This has led to checklists being widely circulated, which are often completed by parents, teachers or professionals who have not been trained in the use of either diagnostic manual. This is also the case with questionnaires. We know from epidemiology that questionnaire or checklist completion alone leads to apparently high rates (up to $14 \%$ ) for hyperactivity disorders in schoolchildren. ${ }^{7}$ This is even higher if parents or teachers rather than both are questioned. The proper use of a checklist approach to diagnosis requires training in judgment of severity, consideration of alternative causes or conditions, evaluation of pervasiveness and impact, and direct clinical evaluation of the child. Under such conditions, good reliability can be obtained. Nevertheless, the pattern of behaviours thought to be indicative of a primary hyperactivity disorder is common among schoolchildren: about $4 \%$ for ADHD and $1 \%$ for HD. ${ }^{8}$

One snag of checklist approaches is that untrained people overlook the possibility that list criteria can be met by children with fundamental psychopathology that is different from primary hyperactivity. Children with general learning disability (mental retardation), generalised anxiety disorder, disinhibited attachment disorder or mania may all fulfil list criteria and lead to fruitless arguments with parents or clinicians who believe that the presence of a certain number of clinical features is sufficient evidence for a primary hyperactivity disorder. 


\section{Coexisting clinical disorders}

ADHD is probably a behavioural syndrome with more than one cause or biological substrate, but HD might be more homogeneous because of its more stringent diagnostic criteria. Because HD research criteria are still new, very little work has been done using HD to define a sample. Most work has used the broader DSM criteria, often thought to identify a heterogeneous group. Indeed, ADHD can be subclassified as predominantly inattentive, predominantly hyperactive-impulsive, and combined-type. Within the latter some workers now think that ADHD coexisting with aggression and with anxiety are different. ${ }^{9}$

There is also the problem that hyperactivity disorders have very high rates of comorbidity. Oppositional defiant disorder or conduct disorder (one cannot have both) is present in most children with $\mathrm{ADHD}, 20 \%$ have coexistent mood disorder, $25 \%$ coexistent anxiety disorder, and $20 \%$ specific developmental disorders such as dyslexia or dyspraxia. ${ }^{9}$ Hyperactivity is also quite common in pervasive developmental disorders such as autism but diagnostic rules preclude the double diagnosis, autism takes precedence. Many children with Tourette's syndrome also fulfil ADHD criteria. The sheer number of comorbid patterns of mood, behaviour, and development could indicate that $\mathrm{ADHD} / \mathrm{HD}$ is a serious psychological vulnerability or that it is a common result of disturbances and developmental delays that disrupt higher cerebral control. On balance, the former seems more likely. Most children with dyslexia, for instance, do not fulfil criteria for ADHD. The clinical issue is that assessment must consider comorbid conditions, that these are not necessarily "competing" diagnoses, and that management needs to address the whole child.

\section{Is a primary hyperactivity disorder qualitatively distinct?}

The components of ADHD or HD are all traits distributed widely in the general child population, and whether primary disorders of hyperactivity are pathological deviants or covarying extremes of such traits is much discussed. Recourse to organic indicators is the logical course but these cannot yet answer the question. Structural and functional brain imaging studies have tended to use small samples within a narrow age range often with broadly defined controls. Not surprisingly, they produce a variety of findings yet with a convergence towards abnormalities in the frontostriatal areas. Anatomically, a smaller right prefrontal cortex and smaller or loss of normal asymmetry in basal ganglia are replicated findings, but not consistently in all studies, probably for technical reasons. Functional imaging using several techniques has not resulted in a single finding, and findings using different techniques are somewhat contradictory, although they generally emphasise reduced activation of the right hemisphere, left frontal, and striatum. ${ }^{10}$ We are a long way from using imaging or quantitative EEG as a clinical diagnostic investigation, and the answer to the question whether primary disorders of hyperactivity represent quantitative or qualitative deviance is not available.

Genetic studies have established beyond doubt that the components of hyperactivity disorders are inherited and powerfully so ${ }^{11}$ although possibly not in girls as there is no overrepresentation of hyperactivity in the families of affected girls. ${ }^{12}$ Whether a single gene or a polygenic model is responsible for disorder is unresolved, but recent evidence from a large study favours a single gene. ${ }^{13}$ However transmitted, the key flaw is thought to be within the dopamine system, either the dopamine transporter gene (DAT1) or the D4 dopamine receptor gene (DRD4). Several studies have indicated abnormalities in these genes in children who have ADHD, but there have also been failed replications so that no finding stands unchallenged. ${ }^{10}$ Possibly the D4 abnormality relates to treatment responsiveness. Once again, there is not enough information to decide whether we are dealing with a category based on qualitative difference from normality or with extreme variation of common cognitive and behavioural traits.

Inheritance studies indicate that not all the variance is accounted for by genes. Shared environmental effects $\left(\chi^{2}\right)$ appear to account for about $15 \%$ of the variance. In the most tightly conducted studies, factors that seem important are maternal depression and social disadvantage,${ }^{14}$ but there are clues that very low birth weight is a determinant. Older work tended to confuse aggressive or other disruptive behaviour with hyperactivity, the two topics frequently coexisting but having somewhat different correlates. Consequently there has been some historical dispute as to whether discordant family relationships are a cause of primary hyperactivity disorders. Taylor et al's influential study indicated that their relevance is in maintaining hyperactivity rather than causing it in the first place. ${ }^{14}$

There is also the likelihood that identifiable developmental pathology, such as fragile $\mathrm{X}$ or hemiplegic cerebral palsy, increases the risk for hyperactivity disorders and therefore need detection in a child presenting initially with behavioural problems.

\section{Essentials of assessment}

It is apparent that the diagnosis cannot be made simply by completing a checklist on information from parents. Information from the school is always required, the child should be examined (although the child may not be hyperactive in a brief outpatient assessment in which he or she is not stressed cognitively), alternative diagnoses must be considered and comorbid and underlying conditions detected. Many cases will need psychometric and educational evaluation. Physical investigations and psychological tests of sustained attention or response inhibition are not yet developed to clinical practice standards. This means that clinical evaluation should be painstaking as there is no objective correlate. Various questionnaires and checklists are available but great caution should be applied to the use of single cut off scores. ${ }^{15} \mathrm{~A}$ single outpatient visit is unlikely to be sufficient to make the diagnosis.

\section{Treatment strategies}

Stimulant medication is the most powerful means of symptomatic control. There is a massive literature (over 1000 references) to the effect that it undoubtedly improves cognition, overactive behaviour, and impulsiveness, ${ }^{16}$ effects most obvious in structured, cognitively demanding settings. In other words classroom behaviour and performance will improve more than home behaviour; teacher report is more sensitive than parental account. It should not, or cannot, always be implemented. Not all children with ADHD need it (although most with HD will), and up to a half of those with the DSM diagnosis can be managed with behavioural or dietary methods. There is little to choose between methylphenidate and dexamphetamine, but experience is greater with the former and a common view is that adverse reactions are a little more common with dexamphetamine. The duration of each dose is short, about three hours, which means that schools have to cooperate with storing and administering a controlled drug. Some parents and teachers (and doctors) are averse to psychotropic medication and will not comply with medical recommendations.

A response to stimulants is not evidence for diagnosis; some "normal" children show similar benefits ${ }^{17}$ and a few children with an undoubted primary hyperactivity disorder do not respond. Some children develop adverse effects: dysphoria, perseverative behaviours, growth retardation, 
auditory hallucinations, stomach pains, and tics. Sometimes these resolve with dose reduction or persistence for a few months. Simple tics and well controlled epilepsy are not absolute contraindications, but indicate the need for cautious monitoring. The liver toxicity that prompted the recent withdrawal of pemoline in the $\mathrm{UK}$ is not an issue with methylphenidate or dexamphetamine, although scare stories on the internet frighten some parents. Very rarely, marrow aplasia can develop but it is far from clear that routine blood counts can predict this and most clinicians do not do them. Addiction is not a problem. Although growth retardation is an occasional problem, ${ }^{16}$ adolescent height was found to be no less in children previously treated with stimulants in one study. ${ }^{18}$ It is quite possible that children with restricted growth are vulnerable, although slowing in growth can happen to any treated child and it is proper practice to monitor growth; repeat prescription without regular personal physical examination is unacceptable. Equivocal evidence for benefit from drug holidays ${ }^{16}$ suggests they are discretionary rather than compulsory. There is often no real need to prescribe on days when a child is not at school as the major benefit is in the classroom setting.

The general consensus is that stimulant medication is insufficient alone yet provides a window of opportunity for more general environmental influences to promote development without being ignored or perverted by the child's condition. Multimodal intervention is the orthodoxy. ${ }^{18} \mathrm{On}$ stimulants, children can learn better, provoke their parents less, and be better tolerated by their peers. ${ }^{19}{ }^{20}$ Treating the child improves their social environment and allows them to benefit from this. Putting things paradoxically, medication is ultimately an environmental approach.

Children who cannot benefit from or tolerate stimulants may be prescribed other medications. ${ }^{21}$ Tricyclic antidepressants (imipramine, nortriptyline, desipramine) and clonidine are effective, mainly on behaviour rather than cognition, but have the potential for cardiac toxicity. Regular cardiovascular system and ECG appraisal is good practice, but imipramine has been so widely prescribed as a symptomatic treatment for enuresis without excessive mortality that ECG monitoring for daily doses of $100 \mathrm{mg}$ or less is probably wasteful. A combination of daytime stimulant and evening clonidine is quite popular as a way of avoiding the sleep onset insomnia common with stimulants, but its long term safety is not established. Adding risperidone to stimulants is favoured by a few enthusiasts and can be dramatically effective in controlling aggressive behaviour but again there are safety concerns and no systematic evidence for benefit. Problems with weight gain, galactorrhoea, and waning efficacy over months are the main limitations. It is not completely free from long term neurological complications, and it is frankly shocking to see that some practitioners use it freely with young children. There have been trials showing benefit for other agents including buspirone, bupropion, venlafaxine, or amantadine but the deployment of these should be reserved to specialist centres. The scientific evidence for benefit from SSRIs in childhood is negligible and there is probably no appreciable benefit from caffeine.

It is generally believed that medication is best avoided in preschool children, partly because of the difficulty in making the diagnosis and the unpredictable effects of stimulants in this age group. Most hyperactive children can be weaned off medication in their mid-teens, although there is a growing assumption in North America that medication should optimally be continued long term, perhaps for life. Existing evidence neither confirms nor denies this. The existence, characteristic presentation, and treatment of so called adult ADHD remains controversial;

\section{Key messages}

- The pattern of restless, impulsive, inattentive behaviour called hyperactivity has not changed since the adoption of the term "attention deficit", which is itself potentially misleading

- Diagnosis remains clinical and there are no objective tests. Assessment must be thorough because the hyperactive pattern of behaviour can be mimicked by other conditions and there is extensive comorbidity. Excessive reliance on checklists is hazardous

- Treatment often, but not always, pivots on stimulant medication. It should be multimodal, implemented early, and benefit the child rather than the caregivers

- The child's school must be involved in assessment and treatment

however, I think it is clear that some affected children will not shed their primary disability at the age of 18 . Continuation of comorbid problems is probably the greatest handicap. The best prospective study ${ }^{22}$ suggests about $10 \%$ of affected boys will still have the ADHD picture in their mid-20s but there was no independent witness account of the young men's adjustment. The authors have said informally that if this information had been available it might have doubled the rate to $20 \%$. There is no scientific evidence for claims that up to $70 \%$ of affected children will still have ADHD as adults but figures like this are bandied around. Residual problems in adult life with self organisation and emotional lability, short of disorder status, seem not uncommon on the basis of clinical observation. The field of adult ADHD is replete with anecdote.

Diet is effective but needs to be stringent if it is to work. Cutting out the "E numbers" is useless, as is the Feingold diet or sugar exclusion. Few-foods (oligo-antigenic) diets can benefit some children ${ }^{23}$ but parents can usually identify provocative foods. The concept of an allergic basis for food intolerance is an ideology. In general diets have only moderate potency and benefit fewer children compared with medication. They are important when families reject medication as an intervention.

Although traditional psychotherapy is useless and family therapy as a sole treatment is no help, behavioural measures should always accompany medication and diet. They can range from straightforward advice to parents to formal behavioural interventions based on recognition of exacerbating stimuli and contingency management. ${ }^{24}$ Parental attitudes may need to be amended when they are based on mistaken assumptions, therefore, the provision of information about hyperactivity is important. This also applies to teachers who as a group have not been well served by informative literature. Purely cognitive approaches do not seem promising; children can learn self instruction to concentrate but do not use it "in vivo".

Most children with primary hyperactivity disorders will have special educational needs as the condition undermines their capacity to learn. They will require individual education plans and some will need a full statement. Once again this underlines the importance of involving schools and educational psychologists in assessment and management. The conventional mainstream classroom is poorly suited to the needs of children with ADHD who are both distractible and disruptive in group work, do not readily attend to instructions given to the class as a whole, require frequent individual feedback, have severe difficulties with cognitive tasks unless these are "chunked" into small steps, and are excitable in response to exasperated teachers. Matters are often worse in secondary schools because of the 
demands that pupils be adept at self organisation. Structure, the assignment of brief tasks only, formal control of classroom activity, a calm orderly atmosphere, and clearly stated expectations are essential. Detailed guidance for teachers on how to manage children with ADHD in schools is available. ${ }^{25} 26$

Educational failure and the emergence of comorbid antisocial behaviour are major long term consequences, and the current debate as to how much inattentive, impulsive restlessness persists into adult life is of less importance than the recognition that long term social failure is extremely common. With this in mind it seems only logical to aim for early recognition and active multimodal treatment. This is for the child's benefit, not the caregivers.

Child and Adolescent Psychiatry,

St George's Hospital Medical School,

London SW17 ORE, UK

1 Still GF. The Goulstonian lectures on some abnormal physical conditions in children. Lancet 1902;i:1008-12.

2 Bradley C. The behavior of children receiving Benzedrine. Am f Psychiatry 1937;94:577-85.

3 Van der Meere JJ, The role of attention. In: Sandberg S, ed. Hyperactivity disorders of childhood. Cambridge: Cambridge University Press, 1996:111-48.

4 Pennington BF, Ozonoff S. Executive functions and developmental psychopathology. F Child Psychol Psychiatry 1996;37:51-87.

5 Sonuga-Barke E, Taylor E, Sembi S, Smith J. Hyperactivity and delay aversion-I. The effect of delay on choice. F Child Psychol Psychiatry 1992; 33:387-98.

6 Oosterlain J, Logan GD, Sergeant JA. Response inhibition in AD/HD, CD comorbid $\mathrm{AD} / \mathrm{HD}+\mathrm{CD}$, anxious and control children: a meta-analysis of studies with the stop task. F Child Psychol Psychiatry 1998;39:411-25.

7 Szatmari P, Offord DR, Boyle MH. Ontario child health study: prevalence of attention deficit disorder with hyperactivity. F Child Psychol Psychiatry 1989;30:219-30.

8 Buitelaar JK, van Engeland H. Epidemiological approaches. In: Sandberg S, ed. Hyperactivity disorders of childhood. Cambridge: Cambridge University Press, 1996:26-68.
9 Jensen PS, Martin BA, Cantwell DP. Comorbidity in ADHD: implications for research, practice, and DSM-IV. $\mathcal{f}$ Am Acad Child Adolesc Psychiatry 1997;36:1065-79.

10 Tannock R. Attention deficit hyperactivity disorder: advances in cognitive, neurobiological and genetic research. $\mathcal{F}$ Child Psychol Psychiatry 1998;39: 65-99.

11 Goodman R, Stevenson J. A twin study of hyperactivity-II. The aetiological role of genes, family relationships and perinatal adversity. $\mathcal{F}$ Child Psychol Psychiatry 1989;30:691-709.

12 Heptinstall E, Taylor E. Sex differences and their significance. In: Sandberg $\mathrm{S}$, ed. Hyperactivity disorders of childhood. Cambridge: Cambridge University Press, 1996:329-49.

13 Faraone SV, Biederman J. Genetics of attention-deficit hyperactivity disorder. In: Greenhill LL, ed. Child and adolescent psychiatric clinics of North America: disruptive disorders. 1994;3:285-301.

14 Taylor E, Sandberg S, Thorley G, Giles S. The epidemiology of childhood hyperactivity. Maudsley Monograph. London: Oxford University Press, 1991

15 Cameron M, Hill P. Recognising hyperactivity: a guide for the cautious clinician. Association for Child Psychology and Psychiatry News. [In press.]

16 Wilens TE, Biederman J. The stimulants. Pediatr Psychopharmacol 1992;15: 191-222.

17 Rapoport JL, Buchsbaum MS, Zahn TP, et al. Dextroamphetamine: cognitive and behavioral effects in normal prepubertal boys. Science 1978;199: $560-2$.

18 Ialongo NS, Horn WF, Pascoe JM, et al. The effects of a multimodal intervention with attention-deficit hyperactivity disorder children: a 9-month follow-up. $\mathcal{F}$ Am Acad Child Adolesc Psychiatry 1993;32:182-9.

19 Barkley R. Hyperactive girls and boys: stimulant drug effects on mother-child interactions. F Child Psychol Psychiatry 1989;30:379-90.

20 Whalen CK. Does stimulant medication improve the peer status of hyperactive children? f Consult Clin Psychol 1989;57:545-9.

21 Spencer T, Biederman J, Wilens T, et al. Pharmacotherapy of attention deficit hyperactivity disorder across the life cycle. $\mathcal{F}$ Am Acad Child Adolesc Psychiatry 1996;35:409-32.

22 Mannuzza S, Klein RG, Bessler A, Malloy P, LaPadula MA. Adult outcome of hyperactive boys. Arch Gen Psychiatry 1993;50:565-76.

23 Carter CM, Urbanowitz M, Hemsley R, et al. Effects of a few-food diet in attention deficit disorder. Arch Dis Child 1993;69:564-8.

24 Schachar R, Tannock R, Cunningham C. Treatment. Sandberg S, ed. Hyperactivity disorders of childhood. Cambridge: Cambridge University Press, 1996:449-76.

25 Cooper P, Ideus K. Attention deficit/hyperactivity disorder: a practical guide for teachers. London, David Fulton Publishers, 1996.

26 DuPaul G, Stoner G. $A D / H D$ in the schools: assessment and intervention strategies. New York: Guilford Press, 1994.

\section{Albumin: saint or sinner?}

The choice of colloid or crystalloid solutions in adult and paediatric resuscitation has been the subject of ongoing debate for many years. A systematic review recently published in the $B M \mathcal{F}$ has again brought this debate to the fore, by indicating that patients treated with human albumin solution (HAS) may have an increased mortality. ${ }^{1}$ The review examined the use of HAS for its licensed indications: hypoalbuminaemia; hypovolaemia from trauma or surgery; and burns. On closer analysis, it is evident that the main aim of many of the studies included was to compare haemodynamic variables and the effects of HAS on nutritive status, rather than to assess the effects of HAS on mortality. While two of the included studies evaluated HAS use in preterm infants, only one assessed HAS in sepsis and none evaluated treatment of critically ill children with HAS. The authors of the systematic review concluded that the use of HAS was associated with a $6 \%$ excess mortality, and that HAS use in critically ill patients should be reviewed. An expert working party is considering the implications of this data.

Where does this leave the practising clinician in the emergency management of the seriously ill or injured child?

The most common cause of childhood mortality worldwide is hypovolaemia, either from gastroenteritis and dehydration, sepsis or trauma. For patients with shock from any cause, treatment of the underlying cause is mandatory. Rapid intravascular volume expansion guided by clinical examination and urine output is frequently adequate to restore organ perfusion and blood pressure. In paediatric septic shock, Carcillo et al showed that fluid resuscitation, of at least $40 \mathrm{ml} / \mathrm{kg}$ in the first hour after presentation, was associated with improved survival without increased risk of pulmonary oedema or the acute respiratory distress syndrome. ${ }^{2}$

\section{What fluid is available}

Decisions regarding which fluid should be used for resuscitation of hypovolaemic children should consider the type of fluid lost, maintenance of plasma oncotic pressure, risk of infection, and cost. Less than $25 \%$ of administered crystalloid solution remains within the intravascular space in normal conditions. The remainder rapidly and freely fills the interstitial and intracellular fluid compartments. ${ }^{3-5}$ Therefore, four times the volume of crystalloid solution would be required to have an equivalent volume enhancing effect of a substance that remained wholly intravascularly.

Albumin is a plasma protein that provides approximately $80 \%$ of intravascular colloid oncotic pressure in normal subjects. Its molecular weight (MW) is 69000 and it is negatively charged at physiological $\mathrm{pH}$. Albumin is relatively impermeable to the endothelial barrier under normal conditions, where its intravascular half life is 24 hours, with haemodynamic improvement persisting up to 36 hours following administration. ${ }^{6}$ Albumin has the additional advantage that it is a physiological protein with many other functions including protein, hormone, and drug binding.

Maintaining plasma colloid oncotic pressure appears to be important in control of normal organ function. Studies in adults have found strong correlations between decreased plasma colloid oncotic pressure (caused by leakage of 
plasma colloids) and the subsequent development and severity of pulmonary oedema. ${ }^{78}$

Synthetic colloids are cheaper than HAS and without the theoretical risk of infectious complications. They are either gelatin or starch derivatives. The gelatin derived compounds (such as Haemaccel (Hoechst Marion Roussel, Middlesex, UK) and Gelofusine (B Braun Medical Ltd, Bucks, UK)) contain various sized molecules (MW 500050 000, mean 35 000), which do not have much benefit over crystalloids in conditions of increased vascular permeability. ${ }^{9}$ The starch based compounds (such as Pentastarch and Hetastarch (Geistlich Sons Ltd, Cheshire, $\mathrm{UK}$ )) while larger than albumin (approximate MW 250 000), expand the plasma volume to a similar degree. ${ }^{40-12}$ All the artificial colloids cause adverse effects such as haemostatic abnormalities and anaphylaxis. ${ }^{12}$ This risk is increased by the use of larger volumes. ${ }^{13}$

\section{Treatment of hypovolaemia}

For hypovolaemia caused by dehydration, the estimated fluid and electrolyte deficits should be replaced with crystalloid. For trauma, effective circulating volume is decreased because of blood loss and reduction in interstitial fluid volume. Although several types of fluid have been suggested for trauma resuscitation, almost any fluid is suitable for initial resuscitation. However, there is no substitute for blood, particularly if the child does not respond to the initial fluid bolus. ${ }^{1314}$

\section{Treatment of sepsis}

The endothelial barrier is disrupted in sepsis causing a capillary leak of plasma proteins and water into the extravascular compartment. Initially this protein leak appears to be size and charge dependent: smaller molecules leak more and negatively charged molecules are preferentially retained within the intravascular compartment. ${ }^{15}$ However, as endothelial dysfunction progresses molecules of all sizes leak. In theory the consequences of large amounts of extravascular protein or any other osmotically active material, are increased extravascular water leading to tissue oedema, persisting until the extravascular colloid is removed or degraded.

Our clinical experience with use of $4.5 \%$ HAS as first line resuscitation fluid in septic shock indicates that it is an effective treatment with no evidence to suggest that it is associated with excess mortality.

Meningococcal septicaemia causes a profound capillary leak syndrome together with myocardial dysfunction and multisystem failure. ${ }^{16}$ Pulmonary oedema occurs in up to $20 \%$ of children with meningococcal sepsis because of the capillary leak and is often present even before any fluid resuscitation.

We have cared for 410 children with meningococcal disease in the past six years. The median volume of fluid resuscitation they required to restore circulating volume was $80 \mathrm{ml} / \mathrm{kg}$ (range 20-300) in the first 12 hours of admission. The vast majority of this fluid was composed of $4.5 \%$ HAS. Despite the use of such large volumes of $4.5 \%$ HAS, we have had a lower than predicted case fatality ratio. ${ }^{17}$ In addition, no child has died on our unit from pulmonary oedema secondary to meningococcal disease since 1993. While this was not a properly conducted study, the fact that mortality in our series of children with meningococcal disease is lower than any of the published figures does not suggest that $4.5 \%$ HAS is dangerous in this condition.

\section{Conclusions}

Studies that have compared colloid (both natural and artificial) and crystalloid solutions for resuscitation have found little difference between them despite the theoretical argu- ments put forward above. ${ }^{18-20}$ No study has specifically evaluated the optimal resuscitation fluid for children or adults with sepsis, and none has examined the use of large volumes of artificial colloids. Therefore, there are no data to guide us on the rational choice of resuscitation fluid for children with sepsis. Until properly conducted, controlled trials are carried out that compare resuscitation fluids in children with sepsis, $4.5 \%$ HAS should remain the first choice for this condition despite its cost and theoretical risks of transmission of infective agents. In addition, controlled studies will be required for other indications where fluid resuscitation is felt necessary to compare the use of crystalloids, artificial colloids, and albumin.

SIMON NADEL

Consultant in Paediatric Intensive Care,

CLAUDINE DE MUNTER

Consultant in Paediatric Intensive Care,

JOSEPH BRITTO

Consultant in Paediatric Intensive Care,

MICHAEL LEVIN

Professor of Paediatrics,

PARVIZ HABIB

Senior Lecturer in Paediatric Intensive Care,

St Mary's Hospital,

Praed Street, London W2 1NY, UK

Correspondence to: Dr Nadel, email: s.nadel@ic.ac.uk

1 Cochrane Injuries Group Albumin Reviewers. Human albumin administration in critically ill patients: systematic review of randomised controlled trials. BMF 1998;317:235-40.

2 Carcillo JA, Davis AL, Zaritsky A. Role of early fluid resuscitation in pediatric septic shock. FAMA 1991;266:1242-5.

3 Rackow EC, Falk JL, Fein IA, et al. Fluid resuscitation in circulatory shock: a comparison of the cardiorespiratory effects of albumin, hetastarch, and a comparison of the cardiorespiratory effects of albumin, hetastarch, and
saline solutions in patients with hypovolemic and septic shock. Crit Care Med 1983;11:839-50.

4 Griffel MI, Kaufman BS. Pharmacology of colloids and crystalloids. Crit Care Clin 1992;8:235-53.

5 Shoemaker WC, Schluter M, Hopkins JA, Appel PL, Schwartz S, Chang PC. Comparison of the relative effectiveness of colloids and crystalloids in the emergency resuscitation. Am f Surg 1981;142:73-81.

6 Rothschild MA, Bauman A, Yalow RS, et al. Tissue distribution of I-13 labelled human serum albumin following intravenous administration. $\mathcal{F}$ Clin Invest 1955;34:1354.

7 Rackow EC, Fein IA, Siegel J. The relationship of the colloid osmoticpulmonary artery wedge pressure gradient to pulmonary edema and mortality in critically ill patients. Chest 1982;82:433-7.

8 Morissette M, Weil MH, Shubin H. Reduction in colloid osmotic pressure associated with fatal progression of cardiopulmonary failure. Crit Care Med 1975;3:115-17.

9 Saddler JM, Horsey PJ. The new generation gelatins. Anaesthesia 1987;42:998-1004.

10 Nagy KK, Davis J, Duda J, Fildes J, Roberts R, Barrett J. A comparison of pentastarch and lactated Ringer's solution in the resuscitation of patients with hemorrhagic shock. Circ Shock 1993;40:289-94.

11 Rackow EC, Mecher C, Astiz ME, Griffel M, Falk JL, Weil MH. Effects of pentastarch and albumin infusion on cardiorespiratory function and coagulation in patients with severe sepsis and systemic hypoperfusion. Crit Care Med 1989;17:394-8.

12 Salmon JB, Mythen MG. Pharmacology and physiology of colloids. Blood Rev 1993;7:114-20.

13 Tobias JD, Rasmussen GE, Yaster M. Multiple trauma in the pediatric patient. In: Rogers MC, ed. Textbook of pediatric intensive care. 3rd ed. Baltimore: Williams and Wilkins, 1996:1467-503.

14 Advanced Trauma Life Support Student Manual. 5th Ed. Chicago: American College of Surgeons, 1995.

15 Nadel S, Kydd P, Hughes C, Levin M. Proteinuria in meningococcal infection: a clue to the capillary leak [abstract]. Clinical Infection Society infection: a clue to the

16 Nadel S, Levin M, Habibi P. Treatment of meningococcal disease in childhood. In: Cartwright K, ed. Meningococcal disease. Chichester: John Wiley and Sons Ltd, 1995:207-43.

17 Levin M, Galassini R, De Munter C, et al, and the Meningococcal Study Group. Improved survival in children admitted to intensive care with meningococcal disease. Proceedings of the 2nd Annual Meeting of the Royal College of Paediatrics and Child Health, York, 1998.

18 So KW, Fok TF, Ng PC, Wong WW, Cheung KL. Randomised controlled trial of colloid or crystalloid in hypotensive preterm infants. Arch Dis Child 1997;76:F43-6.

19 Pockaj BA, Yang JC, Lotze MT, et al. A prospective randomized trial evaluating colloid versus crystalloid resuscitation in the treatment of the vascular leak syndrome associated with interleukin-2 therapy. Immunotherapy 1994; 15:22-8.

20 Nagy KK, Davis J, Duda J, Fildes J, Roberts R, Barrett J. A comparison of pentastarch and lactated Ringer's solution in the resuscitation of patients with hemorrhagic shock. Circ Shock 1993;40:289-94. 